\title{
Exponential Stabilization of Delay Neutral Systems under Sampled-Data Control
}

\author{
Alexandre Seuret, Emilia Fridman and Jean-Pierre Richard
}

\begin{abstract}
This paper considers the exponential stabilization of delay systems of the neutral type via sampled-data control. The control input of the neutral system can present a delay, constant or variable. The sampling period is not necessarily constant. It is only assumed that the time between to successive sampling instants is bounded. Since the sampling effect (sampling and zero-holder) is equivalent to a variable delay, the resulting system is modelled as a continuous-time one, where the control input has a 'non-small' time-varying delay belonging to some interval $[h-\mu, h+\mu]$. For instance, $h-\mu$ may represent the minimum input delay, and $2 \mu$ the additional delay generated by the combination of the sampling effect with the input delay variation. This results in a system with 'nonsmall' time-varying delays (i.e. delays with a known and nonzero minimum value), the exponential stabilization of which is possible under LMI conditions. Two examples are provided. The first one deals with the sampled-data control of a neutral system. The second one considers the stabilization of a flexible rod with continuous, delayed control
\end{abstract}

Index Terms - Time-varying delay, neutral system, sampleddata control, stabilization, LMI, flexible rod.

\section{INTRODUCTION}

Recent papers [8], [27] considered the modelling of continuous-time systems with sampled-data control in the form of continuous-time systems with delayed control input and which model was combined with Lyapunov-based methods. The digital control law produced by a sampler with zero-holder can be represented as follows:

$$
\begin{gathered}
u(t)=u_{d}\left(t_{k}\right)=u_{d}\left(t-\left(t-t_{k}\right)\right)=u_{d}(t-\eta(t)), \\
t_{k} \leq t<t_{k+1}, \eta(t)=t-t_{k} .
\end{gathered}
$$

Here, $u_{d}$ is the discrete-time control signal and the timevarying delay $\eta(t)=t-t_{k}$ is piecewise-linear with derivative $\dot{\eta}(t)=1$ for $t \neq t_{k}$. Moreover, $\eta(t) \leq t_{k+1}-t_{k} \leq \mu_{1}$, where $\mu_{1}$ is the maximum sampling interval. This case of 'small' time-varying delay $\tau(t) \in[0, \mu]$ has been analyzed in the above papers by using Lyapunov-Krasovskii method via the descriptor model transformation [6] and by the Lyapunov-Razumikhin technique, correspondingly.

If there is an additional constant delay $h_{2}>0$ in the control input, the delayed digital control law can be modelled

A. Seuret and J-P. Richard are with LAGIS CNRS UMR 8146, Ecole Centrale de Lille, BP 48, 59651 Villeneuve d'Ascq Cedex, France seuret.alexandredec-lille.fr and jean-pierre.richard@ec-lille.fr

E. Fridman is with Department of Electrical Engineering, Tel-Aviv University, Tel-Aviv 69978, Israel emilia@eng.tau.ac.il in the form:

$$
\begin{aligned}
u\left(t-h_{2}\right) & =u_{d}\left(t_{k}-h_{2}\right)=u_{d}\left(t-h_{2}-\left(t-t_{k}\right)\right), \\
u\left(t-h_{2}\right) & =u_{d}(t-\tau(t)), \\
t_{k} & \leq t<t_{k+1}, \quad \tau(t)=h_{2}+t-t_{k} .
\end{aligned}
$$

Thus, the delay is 'non-small', i.e. $\tau(t) \in\left[h_{2}-\mu_{2}, h_{2}+\right.$ $\mu_{2}$ ] with $h_{2}>0$ and $h_{2}-\mu_{2} \geq 0$. Only a few papers [7], [15], [23] have been published on this topic. The asymptotic stability of linear retarded-type systems with one time-varying 'non-small' delay has been analyzed by [15]. Sufficient stability conditions, there, have been derived via a modification of 'complete' Lyapunov-Krassovskii functionals, which corresponds to necessary and sufficient stability conditions. In [7], a new construction of LyapunovKrasovskii functionals, which generalizes the descriptor one [6], was introduced. Stability and $H_{\infty}$ control of neutral systems with multiple 'non-small' delays have been studied in [23], using the same idea.

Concerning the sampled-data stabilization problem, two main approaches have been used before the paper [8] (see e.g. [4], [22], [20], [24]). The first one is based on the lifting technique [1], [26] in which the problem is transformed into an equivalent finite-dimensional discrete problem. This approach seems to be unapplicable to the case of state-delay. The second approach is based on the representation in the form of an hybrid discrete/continuous model. Application of this approach to linear systems leads to necessary and sufficient conditions for stability and $L_{2}$-gain analysis in the form of differential equations (or inequalities) with jumps (see e.g. [3], [22]). The latter approach has been applied to $H_{\infty}$ control of retarded type systems with constant state delay [9], where partial differential Riccati equations with jumps have been derived. The method is not applicable to neutral systems with input delay. Recently, it has been applied to the sampled-data stabilization of linear state-delay systems in the case of uniform (periodic) sampling [14]. To overcome difficulties of solving differential inequalities with jumps, a piecewise-linear in time Lyapunov functional has been suggested. As a result, LMIs have been derived which do not depend on the sampling interval and thus are very conservative.

Concerning the exponential stabilization problem, some applications such as observer design, networked control, tele-operated systems or chained systems often need exponential convergence, since it is the best way of ensuring some speed performance. Some authors have investigated the exponential stability of delayed systems [18], [19]. 
However, these results are limited to constant delays. In many cases, such as the communication lines used in networked control, the delays cannot be reduced to constant ones. Recently, Seuret et al developed exponential stability results for retarded systems with time-varying delays [21].

The present work focuses on exponential stability and stabilization of neutral systems with bounded, time-varying delays. The approach, which involves a Lyapunov-Krasovskii functional and a descriptor model, was developed in [10], [11], [12] for asymptotic stabilization. We combine it with a polytopic approach [25] that allows one to reduce the time-varying delay to a convex sum of its bounds [21].

In the present paper, we generalize the approach of [8] to the sampled-data stabilization of systems with state and input delay. Moreover we consider, more widely, neutral-type linear systems described by: $\dot{x}(t)-F \dot{x}(t-g(t))=A_{0} x(t)+$ $\left.A_{1} x\left(t-\tau_{1}(t)\right)+B u\left(t-h_{2}\right)\right)$, which will be presented in the next section. For systems with $g(t)=h_{1}$ constant, we complete this result with the exponential stabilization. The solutions are derived by solving the problem for a continuous-time system with uncertain but bounded timevarying 'non small' delay in the control input.

The obtained conditions are robust with respect to different samplings with the only requirement that the maximum sampling interval $\mu_{1}$ is not greater than some computed $\mu$. Moreover, the feasibility of the LMI is guaranteed for small $\mu$ if the corresponding continuous-time controller stabilizes the system.

Notation: Throughout the paper, the superscript $T$ stands for matrix transposition, $\mathscr{R}^{n}$ denotes the $n$-dimensional Euclidean space with the norm $|x|$ of vector $x, \mathscr{R}^{n \times m}$ is the set of $n \times m$ real matrices with the Euclidean norm $\|\cdot\|$. The notation $P>0$ for $P \in \mathscr{R}^{n \times n}$ means that $P$ is symmetric and positive definite. A star $*$ in a matrix represents a symmetrical entry.

\section{PROBLEM FORMULATION}

Consider the system:

$$
\begin{aligned}
\dot{x}(t)-F \dot{x}(t-g(t))= & A_{0} x(t)+A_{1} x\left(t-\tau_{1}(t)\right) \\
& +B u\left(t-h_{2}\right), \\
x(t)=\phi(t), \quad \text { for } \quad & t \in[-\bar{h}, 0],
\end{aligned}
$$

where $x(t) \in \mathscr{R}^{n}$ is the system state, $u(t) \in \mathscr{R}^{m}$ is the control input, $A_{i}$ and $B$ are constant matrices, $\phi$ is a continuously differentiable initial function and $\bar{h}$ is an upper-bound on the time-delays $\tau_{1}$ and $g$. For simplicity only, we consider one delay $\tau_{1}$ and one delay $g$. However, the results of this paper can be easily extended to the case of multiple delays $\tau_{1}, \ldots, \tau_{m}, g_{1}, \ldots, g_{k}$

The input delay $h_{2}$ is constant but this also can be easily generalized to $h_{2}(t)$ time-varying, since $h_{2}$ will be considered in combination with an additional varying delay coming from the variable sampling.

We assume that $g(t)$ is a differentiable function satisfying $\dot{g}(t) \leq d_{0}<1$ for all $t \geq 0$, where $d_{0}$ is a known upperbound. Moreover, we assume that $\|F\|<1$. The latter

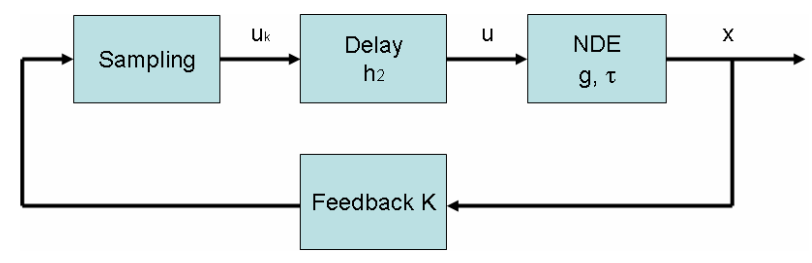

Fig. 1. Problem representation

guarantees that the difference equation $x(t)-F x(t-g(t))=$ 0 is asymptotically stable [2],[16]. Our asymptotic stability results will be independent on $g$ and dependent on $d_{0}$. Our exponential stability results will be considered in the case $\dot{g}(t)=0$.

The uncertain delay $\tau_{1}(t)$ is supposed to have the following form $\tau_{1}(t)=h_{1}+\eta_{1}(t)$, where $h_{1}>0$ is a constant value and $\eta_{1}$ is a time-varying perturbation. We will consider that $\eta_{1}(t)$ is a piecewise-continuous function, satisfying:

$$
-\mu_{1} \leq \eta_{1}(t) \leq \mu_{1}, \quad \forall t \geq 0
$$

We consider a piecewise-constant control law of the form $u\left(t-h_{2}\right)=u_{d}\left(t_{k}-h_{2}\right), t_{k} \leq t<t_{k+1}$, where $u_{d}$ is a discretetime control signal and $0=t_{0}<t_{1}<\ldots<t_{k}<\ldots$ are the sampling instants. Our objective is to find a state-feedback stabilizing controller in the form:

$$
u\left(t-h_{2}\right)=K x\left(t_{k}-h_{2}\right), \quad t_{k} \leq t<t_{k+1} .
$$

The piecewise-constant control law is equivalent to a continuous-time control with a time-varying piecewisecontinuous (continuous from the right) delay $\tau_{2}(t)=h_{2}+$ $t-t_{k}$ as given in (2), where $h=h_{2}$. Thus, we look for a state-feedback controller of the form $u(t)=K x\left(t-\tau_{2}(t)\right)$. Substituting the latter controller into (3), we obtain the following closed-loop system:

$$
\begin{array}{ll}
\dot{x}(t)-F \dot{x}(t-g(t))= & A_{0} x(t)+A_{1} x\left(t-\tau_{1}(t)\right) \\
& +B K x\left(t-\tau_{2}(t)\right) \\
\tau_{2}(t)=h_{2}+t-t_{k}, \quad & t_{k} \leq t<t_{k+1} .
\end{array}
$$

We assume that A1: $\quad-\mu_{2} \leq t_{k+1}-t_{k} \leq \mu_{2}, \forall k \geq 0$.

From A1 and since $\tau_{2}(t)=h_{2}+t_{k+1}-t_{k}$, it follows that $h_{2}-\mu_{2} \leq \tau_{2}(t) \leq h_{2}+\mu_{2}$. We will further consider (6) as the 'system with uncertain and bounded delay'.

\section{ASYMPTOTIC STABILITY OF THE CLOSED-LOOP SYSTEM}

Lemma 1 (Stability, [7] (Case 1)): Given a gain matrix $K$, the system (6) is stable for all the samplings satisfying $\mathrm{A} 1$, if there exist $n \times n$ matrices $0<$ $P_{1}, P_{2}, P_{3}, S_{i}, U, Y_{k 1}, Y_{k 2}, Z_{k 1}, Z_{k 2}, Z_{k 3}, R_{k}$ and $R_{k a}, k=1,2$ 
that satisfy:

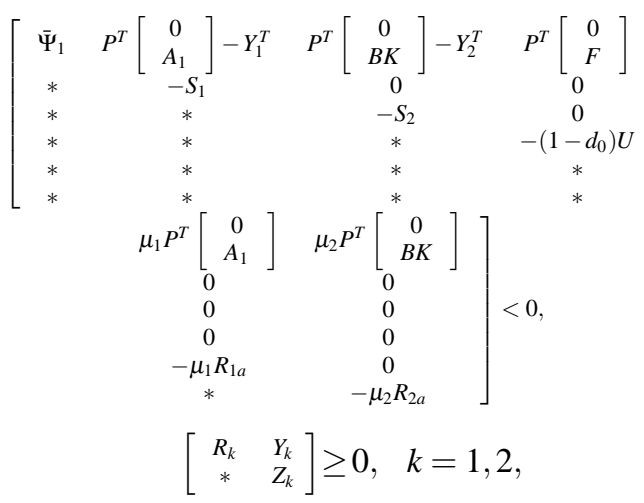

where $Y_{k}, Z_{k}$ and $\bar{\Psi}_{1}$ are given by:

$$
\begin{aligned}
& \bar{\Psi}_{1}=\bar{\Psi}_{n}+\left[\begin{array}{cc}
0 & 0 \\
0 & \sum_{k=1}^{2} 2 \mu_{k} R_{k a}
\end{array}\right], \\
& \bar{\Psi}_{n}=P^{T}\left[\begin{array}{cc}
0 & I \\
A_{0} & -I
\end{array}\right]+\left[\begin{array}{cc}
0 & I \\
A_{0} & -I
\end{array}\right]^{T} P+\sum_{k=1}^{2}\left(h_{k}+\mu_{k}\right) Z_{k} \\
& +\sum_{k=1}^{2}\left[\begin{array}{c}
Y_{k} \\
0
\end{array}\right]+\sum_{k=1}^{2}\left[\begin{array}{c}
Y_{k} \\
0
\end{array}\right]^{T}, \\
& +\left[\begin{array}{cc}
\sum_{k=1}^{2} S_{k} & 0 \\
0 & \sum_{k=1}^{2}\left(h_{k}+\mu_{k}\right) R_{k}+U
\end{array}\right]
\end{aligned}
$$

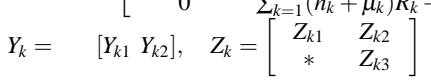

\section{EXPONENTIAL STABILITY OF THE CLOSED-LOOP SYSTEM}

We consider in this section the neutral type system (3) in the (particular but frequent) case $g(t)=h_{1}$. As usual [19], [21], being given some rate $\alpha>0$, the closed-loop system (3) is said to be $\alpha$-stable, or 'exponentially stable with the rate $\alpha$ ', if there exists a scalar $K \geq 1$ such that its solution $x\left(t ; t_{0}, \phi\right)$ satisfies:

$$
\left|x\left(t, t_{0}, \phi\right)\right| \leq K|\phi| e^{-\alpha\left(t-t_{0}\right)} .
$$

Substituting the new variable $z(t)=e^{\alpha t} x(t)$ in (6), we find:

$$
\begin{aligned}
\dot{z}(t)= & \left(A_{0}+\alpha I\right) z(t)+e^{\alpha \tau_{1}} A_{1} z\left(t-\tau_{1}(t)\right) \\
& +e^{\alpha \tau_{2}} B K z\left(t-\tau_{2}(t)\right)+F e^{\alpha t} \dot{x}\left(t-h_{1}\right),
\end{aligned}
$$

the last term of which can be expressed with the variable $z$ :

$$
e^{\alpha t} \dot{x}\left(t-h_{1}\right)=e^{\alpha h_{1}} \dot{z}\left(t-h_{1}\right)-\alpha e^{\alpha h_{1}} z\left(t-h_{1}\right),
$$

which finally leads to the transformed neutral system:

$$
\begin{aligned}
\dot{z}(t)= & \left(A_{0}+\alpha I\right) x(t)+e^{\alpha \tau_{1}(t)} A_{1} x\left(t-\tau_{1}(t)\right) \\
& -\alpha e^{\alpha h_{1}} F z\left(t-h_{1}\right)+F e^{\alpha h_{1}} \dot{z}\left(t-h_{1}\right) \\
& +e^{\alpha \tau_{2}(t)} B K x\left(t-\tau_{2}(t)\right) .
\end{aligned}
$$

Our purpose is to find conditions for the solution $z=0$ of this transformed system (13) to be stable. Then, these conditions will assure the exponential, $\alpha$-stability (10) of the original system (6). Note that a necessary condition of exponential stability is that the spectral radius of $e^{\alpha h_{1}} F$ is less than one.

However, system (13) is a linear time-varying one because of the gains $e^{\alpha \tau_{1}(t)}$ and $e^{\alpha \tau_{2}(t)}$. This does not allow for applying directly Lemma 1 . This difficulty can be overcome by applying a polytopic approach [21] [13]. Indeed, according to A1 and (2), the time-varying terms $e^{\alpha \tau_{1}(t)}$ and $e^{\alpha \tau_{2}(t)}$ are bounded as follows:

$$
e^{\alpha\left(h_{i}-\mu_{i}\right)} \leq e^{\alpha \tau_{i}(t)} \leq e^{\alpha\left(h_{i}+\mu_{i}\right)}, \quad \forall t \geq 0, \quad \forall i=1,2 .
$$

This means there exists unknown scalar and positive functions $\lambda_{i j}: \mathscr{R} \rightarrow \mathscr{R},(i, j) \in\{1,2\}^{2}$, satisfying the following convexity conditions:

$$
\forall t \geq 0, \quad \forall(i, j) \in\{1,2\}^{2} \quad \lambda_{i j}(t) \geq 0, \quad \sum_{i, j=1}^{2} \lambda_{i j}(t)=1
$$

and such that equation (13) is written as:

$$
\begin{aligned}
\dot{z}(t)= & \sum_{i, j=1}^{2} \lambda_{i j}(t)\left\{\left(A_{0}+\alpha I\right) z(t)+\beta_{1 i} A_{1} z\left(t-\tau_{1}(t)\right)\right. \\
& -\alpha e^{\alpha h_{1}} F z\left(t-h_{1}\right)+F e^{\alpha h_{1}} \dot{z}\left(t-h_{1}\right) \\
& \left.+\beta_{2 j} B K z\left(t-\tau_{2}(t)\right)\right\},
\end{aligned}
$$

where

$$
\begin{array}{ll}
\beta_{11}=e^{\alpha\left(h_{1}-\mu_{1}\right)}, & \beta_{12}=e^{\alpha\left(h_{1}+\mu_{1}\right)}, \\
\beta_{21}=e^{\alpha\left(h_{2}-\mu_{2}\right)}, & \beta_{22}=e^{\alpha\left(h_{2}+\mu_{2}\right)},
\end{array}
$$

Now, applying the results of [7] (Case 1) yields the following result.

Theorem 1 (Exponential stability): Given a gain matrix $K$, the system (6) is $\alpha$-stable for all the samplings satisfying A1, if there exist $n \times n$ matrices $0<P_{1}, P_{2}, P_{3}, S_{k}, U$, $Y_{k 1}, Y_{k 2}, Z_{k 1}, Z_{k 2}, Z_{k 3}, R_{k}$ and $R_{k a}, k=1,2$ that satisfy (8) and

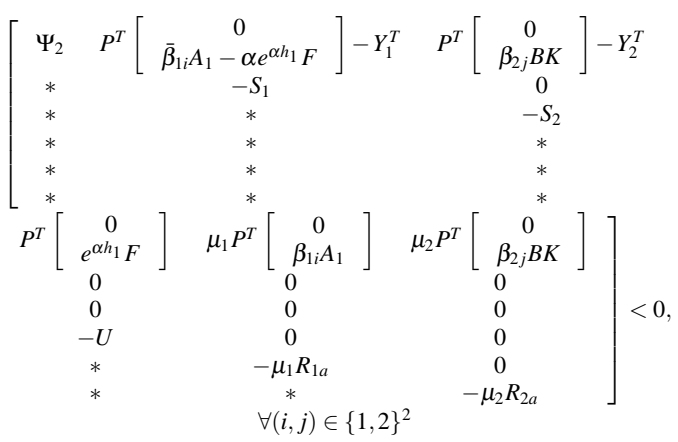

where $Y_{i}, Z_{i}$ are given by (9) and

$$
\begin{aligned}
\Psi_{2}= & \bar{\Psi}_{n e}+\left[\begin{array}{cc}
0 & 0 \\
0 & \sum_{k=1}^{2} 2 \mu_{k} R_{k a}
\end{array}\right], \\
\bar{\Psi}_{n e}= & P^{T}\left[\begin{array}{cc}
0 & I \\
A_{0}+\alpha I & -I
\end{array}\right]+\left[\begin{array}{cc}
0 & I \\
A_{0}+\alpha I & -I
\end{array}\right]^{T} P \\
& +\sum_{k=1}^{2}\left(\left[\begin{array}{c}
Y_{k} \\
0
\end{array}\right]+\left[\begin{array}{c}
Y_{k} \\
0
\end{array}\right]^{T}\right)+\sum_{k=1}^{2}\left(h_{k}+\mu_{k}\right) Z_{k} \\
& +\left[\begin{array}{cc}
\sum_{k=1}^{2} S_{k} & 0 \\
0 & \sum_{k=1}^{2}\left(h_{k}+\mu_{k}\right) R_{k}+U
\end{array}\right],
\end{aligned}
$$

\section{EXPONENTIAL STABILIZATION OF NEUTRAL SYSTEMS}

Theorem 2 (Exponential stabilization): The control law (5) exponentially stabilizes system (3) if, for some positive numbers $\alpha$ and $\varepsilon$, there exists a positive definite matrix $\bar{P}_{1}$, matrices of size $n \times n \bar{P}, \bar{U}, \bar{Z}_{k 1}, \bar{Z}_{k 2}, \bar{Z}_{k 3}, \bar{Y}_{k 1}, \bar{Y}_{k 2}$ from 
definition (9) and a $n \times m$ matrix $W$, such that the following LMI conditions hold for $i, j=1,2$ :

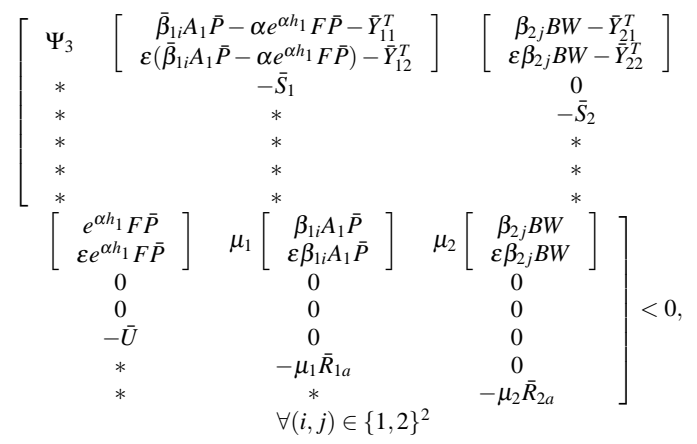

and

$$
\left[\begin{array}{ccc}
\bar{R}_{i} & \bar{Y}_{i 1} & \bar{Y}_{i 2} \\
* & \bar{Z}_{i 1} & \bar{Z}_{i 2} \\
* & * & \bar{Z}_{i 3}
\end{array}\right] \geq 0,
$$

where

$$
\begin{aligned}
\bar{\Psi}_{311}= & \left(A_{0}+\alpha I\right) \bar{P}+\bar{P}^{T}\left(A_{0}+\alpha I\right)^{T} \\
& +\sum_{k=1}^{2}\left(\bar{S}_{k}+\left(h_{k}+\mu_{k}\right) \bar{Z}_{k 1}+\bar{Y}_{k 1}+\bar{Y}_{k 1}^{T}\right), \\
\bar{\Psi}_{312}= & \bar{P}_{1}-\bar{P}+\varepsilon \bar{P}^{T}\left(A_{0}+\alpha I\right)^{T} \\
& +\sum_{k=1}^{2}\left(\left(h_{k}+\mu_{k}\right) \bar{Z}_{k 2}+\bar{Y}_{k 2}\right), \\
& -\varepsilon\left(\bar{P}+\bar{P}^{T}\right) \\
& +\sum_{k=1}^{2}\left(\left(h_{k}+\mu_{k}\right)\left(\bar{Z}_{k 3}+\bar{R}_{k}\right)+2 \mu_{k} \bar{R}_{k a}\right),
\end{aligned}
$$

The corresponding $\alpha$-stabilizing state-feedback gain is given by:

$$
K=W \bar{P}^{-1} .
$$

Proof: Following [23], we apply Theorem 1 with: $P_{3}=\varepsilon P_{2}$, where $\varepsilon \in \mathscr{R}$ is a tuning scalar parameter. Note that $P_{2}$ is nonsingular since the only matrix which can be negative definite in the second block on the diagonal of (19) is $-\varepsilon\left(P_{2}+P_{2}^{T}\right)$. Defining:

$$
\bar{P}=P_{2}^{-1}
$$

For all the matrical variables $V \in\left[\begin{array}{llllll}P_{1} & Y_{i j} & S_{i} & U & R_{i} & R_{i a}\end{array}\right.$ $\left.Z_{i k}\right]$ for all $i=1,2, j=1,2, k=1,2,3$ the new variable $\bar{V}$ is defined by $\bar{P}^{T} V \bar{P}$. and $W=K \bar{P}$, multiplying (19) by $\operatorname{diag}\{\bar{P}, \bar{P}, \bar{P}, \bar{P}, \bar{P}, \bar{P}, \bar{P}\}$, and its transpose, from the right and the left, respectively, and multiplying (8) by $\operatorname{diag}\{\bar{P}, \bar{P}, \bar{P}\}$ and its transpose, from the right and the left, achieves the proof.

Remark 1: In the case $\alpha=0$, Theorem 1 assures that the state-feedback gain $K$ asymptotically stabilizes system (3).

\section{EXAMPLE 1}

Consider the following example, taken from [17]. We address the problem of finding an exponentially stabilizing control for system (3) with the values:

$$
\begin{array}{cc}
A_{0}=\left[\begin{array}{ll}
1 & 0 \\
0 & 1
\end{array}\right], & A_{1}=\left[\begin{array}{cc}
-1 & 0 \\
-1 & -0.9
\end{array}\right], \\
F=\left[\begin{array}{cc}
0.1 & 0 \\
0 & 0.1
\end{array}\right], & B=\left[\begin{array}{l}
0 \\
1
\end{array}\right] .
\end{array}
$$

Solving the LMIs of Theorem 2 for $h_{1}=0.5, \mu_{1}=0.2$, $h_{2}=0.6$, and $\varepsilon=4.2$ leads to the state-feedback gain $K=$ $[0.7670,-0.2241]$ which asymptotically stabilizes $(\alpha=0)$ the system up to $\mu_{2}=0.21$.
Concerning the $\alpha$-stabilization, for $\alpha=1.19, h_{1}=$ $h_{2}=0.16, \mu_{1}=\mu_{2}=0.09$ and $\varepsilon=2.3$, the computed state-feedback gain $K=[1.0215-1.0741]$ exponentially stabilizes the system. This result ensures that the system is exponentially stable with a delayed and nonuniform sampled control. The corresponding simulation results are given on Figure 2.

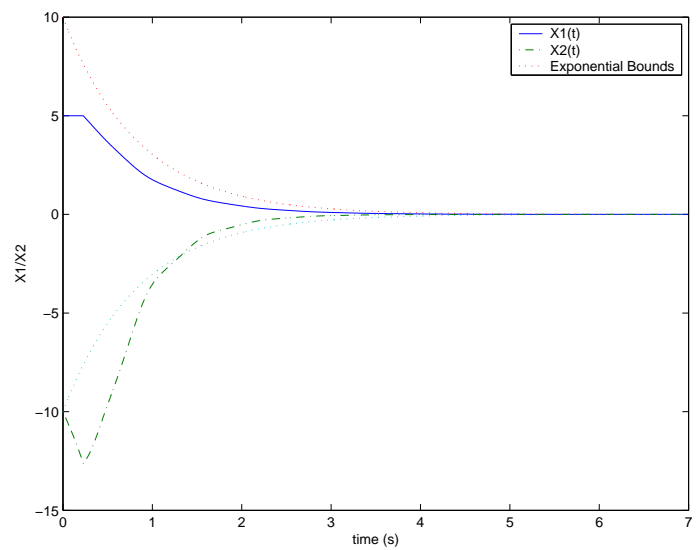

Fig. 2. Simulation of the system for $\alpha=1.19, h_{1}=h_{2}=0.16, \mu_{1}=\mu_{2}=$ 0.09

Note that for larger values $\alpha>1$, Theorem 2 cannot ensure $\alpha$-stability.

\section{EXAMPLE 2}

The second example, of the neutral type, is not concerned with sampled-data control, but it still uses Theorem 2. Several authors interpreted the wave equation describing the torsional behavior of a flexible rod with a mass as a linear system with delayed terms. A neutral state representation for flexible rod equation is given in [5] :

$$
\begin{aligned}
& \dot{x}_{1}(t)=x_{2}(t), \\
& \dot{x}_{2}(t)=\dot{x}_{2}(t-2 T)-x_{2}+x_{2}(t-2 T)+u(t-T),
\end{aligned}
$$

where $T$ represents the delay and depends on the parameters of the system.

In such a neutral case, the difference operator $x(t)-$ $F x(t-g)$ must be stable in sense of Shür-Cohn, which corresponds to formal stability [2]. Then, [2] and [5] introduce a stabilizing control of the form :

$$
u(t)=-\lambda \dot{x}_{2}(t-T)+v(t)
$$

with $\lambda \in] 0,2[$. In [2] and [5], $v(t)$ was designed on the basis of $x(t-T)$ measurement. Here, one suppose that $\dot{x}_{2}(t-T)$ is still measured, but that $x$ is measured with some additional time-varying delay $\mu$, i.e. $x(t-T-\mu(t)$. So the following control law is proposed:

$$
v(t)=K x(t-T-\mu(t)),
$$

where $\mu$ is such that $\|\mu(t)\| \leq \mu_{2}$ and $K$ is a state feedback gain of appropriate dimension. 
Then, flexible rod equations are in the form of (3) with:

$$
\begin{array}{ll}
A_{0}=\left[\begin{array}{cc}
0 & 1 \\
0 & -1
\end{array}\right], & A_{1}=\left[\begin{array}{ll}
0 & 0 \\
0 & 1
\end{array}\right], \\
F=\left[\begin{array}{cc}
0 & 0 \\
0 & 1-\lambda
\end{array}\right], & B=\left[\begin{array}{l}
0 \\
1
\end{array}\right] .
\end{array}
$$

Theorem 2 is adapted to the flexible rod case, with $\lambda=$ $0.2, T=0.1$, by taking $\alpha=1.05, \mu_{1}=0, \mu_{2}=0.08$ and $\varepsilon=$ 1.32. After controlling that $\left\|e^{\alpha h_{1}} F\right\|<1$, the corresponding simulation results are given on Figure 3. They show the expected exponential convergence.

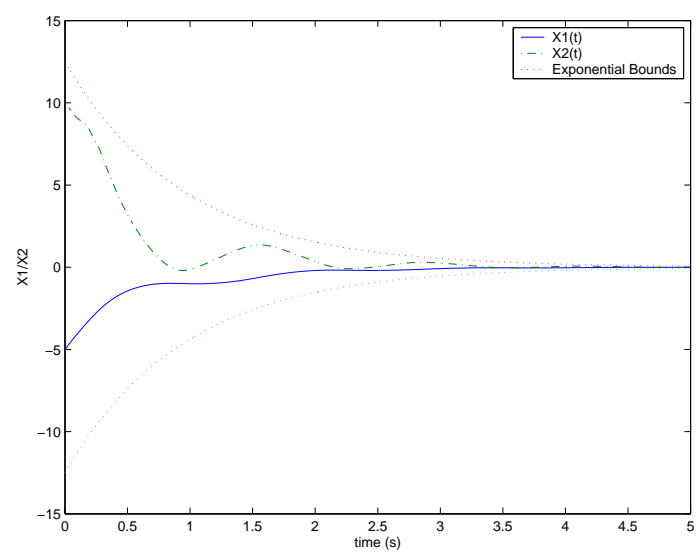

Fig. 3. Simulation of flexible rod with $\alpha=1.05, h_{1}=h_{2}=0.1, \mu_{1}=$ $0 \mu_{2}=0.08$

\section{CONCLUSION}

The obtained results generalize several recent works: [8] and [14] to neutral systems; [7], [8] and [15] to exponential stabilization; [14] to non uniform sampling; [8] to non-small delays; [2] and [5] to delayed measurement..

In order to shorten the presentation, it was only considered one delay $\tau_{1}$, one delay $g$ and a constant input delay $h_{2}$. However, the results of this paper can be easily extended to the case of multiple delays $\tau_{1}, \ldots, \tau_{m}, g_{1}, \ldots, g_{k}$ and of a time-varying $h_{2}(t)$. Another possible extension includes robustness issues.

\section{REFERENCES}

[1] B.A. Bamieh, and J.B Jr Pearson, "A general framework for linear periodic systems with applications to $H^{\infty}$ sampled-data control", IEEE Trans on Automatic Control, vol. 37(4), 1992, pp 265-271.

[2] C.I. Byrnes, M.W. Spong, T.J. Tarn, "A several complex variables approach to feedback stabilization of linear neutral delay-differential systems", Math. Systems Theory, vol. 17, 1984, pp 97-133.

[3] T. Chen and B. Francis, " $\mathrm{H}_{2}$ optimal sampled-data control" IEEE Trans. on Automatic Control, vol. 36, 1991, pp 387-397.

[4] G. Dullerud and K. Glover,"Robust Stabilization of sampled-data systems to structured LTI perturbations", IEEE Trans. on Automatic Control, vol. 38, 1993, pp 1497-1508.

[5] M. Fliess, H. Mounier, P. Rouchon, J. Rudolph, "Controlability and motion planning for linear delay systems with application to flexible rod", Proceedings of the $34^{\text {th }}$ Conference on Decision and Ccontrol New Orleans, LA-December 1995.
[6] E. Fridman,"New Lyapunov-Krasovskii functionals for stability of linear retarded and neutral type systems", System and Control Letters, vol. 43, 2001, pp 309-319.

[7] E. Fridman,"Stability of linear functional differential equations: A new Lyapunov technique", Proceedings of Mathematical Theory of Networks and Systems, Leuven, 2004.

[8] E. Fridman, A. Seuret and J-P. Richard,"Robust sampled-data stabilization of linear systems: An input delay approach", Automatica, vol. 40(8), 2004, pp 1441-1446.

[9] E. Fridman and U. Shaked,"Finite horizon $H^{\infty}$ state-feedback control of continuous-time systems with state delay", IEEE Trans. on Automatic Control, vol. 45, 2000, pp 2406-2411.

[10] E. Fridman and U. Shaked,"An improved Stabilization method for linear time-delay systems", IEEE Trans. on Automatic Control, vol. 47(11), 2002, pp 1931-1937.

[11] E. Fridman and U. Shaked,"Parameter dependent stability and stabilization of uncertain time-delay systems", IEEE Trans. on Automatic Control, vol. 48, 2003, pp 861-866.

[12] E. Fridman, F. Gouaisbaut, M. Dambrine and J-P. Richard,"Sliding mode control of systems with time-varying delays via descriptor approach", Proceedings of ECC'03, Cambridge, 2003.

[13] F. Gouaisbaut, Y. Blanco and J-P. Richard, "Robust control of nonlinear systems with delay : A design via polytopic formulation", International Journal of Control, vol. 77(4), 2002, pp 206-215.

[14] L. Hu, Y. Cao C. Cheng and H. Shao, "Sampled-data Control for time-delay systems", Journal of the Franklin Institute, vol. 339(2), 2002, pp 231-238.

[15] V.-L. Kharitonov and S.-I. Niculescu, "On the stability of linear systems with uncertain delay", IEEE Trans. on Automatic Control, vol. 48(1), 2003, pp 127-132.

[16] V. Kolmanovskii and A. Myshkis, "Applied theory of functional differential equations", Kluwer, 1999.

[17] X. Li and C. de Souza, "Criteria for robust stability and stabilization of uncertain linear systems with state delay", Automatica, vol. 33, 1997, pp 1657-1662.

[18] P-L. Liu, "Robust exponential stabilization for uncertain systems with state and control delay", International Journal of Systems Science, vol. 34, 2003, pp 675-682.

[19] S.I. Niculescu, C. de Souza, L. Dugard and J.M. Dion, "Robust exponential Stability of uncertain systems with time-varying delays", IEEE Trans. on Automatic Control, vol. 43, 1998, pp 743-748.

[20] Y. Oishi, "A bound of conservativeness in sampled-data robust stabilization and its dependence on sampling periods", Systems and Control Letters, vol. 32, 1997, pp 11-19.

[21] A. Seuret, M. Dambrine and J.-P. Richard, "Robust exponential stabilization for systems with time-varying delays", $5^{\text {th }}$ IFAC Workshop on Time-Delay Systems, 2004, Leuven.

[22] N. Sivashankar and T. Khargonekar, "Characterization of the $L_{2}$ introduced norm for linear systems with jumps with applications to sampled-data systems", SIAM J. Control and Optimization, vol. 32, 1994, pp 1128-1150.

[23] V. Suplin, E. Fridman and U. Shaked, " $H^{\infty}$ control of linear uncertain time-delay systems - a projection approach", Proc. of CDC, 2004, Bahamas.

[24] H. Trentelman and A. Stoorvogel, "Sampled-data and discrete-time $H^{2}$ optimal Control", J. Control and Optimization, vol. 48, 2003, pp 861-866.

[25] Y. Xia and Y. Jia, "Robust control of state delayed systems with politopic uncertainties via parameter-dependent Lyapunov functionals", Systems and Control Letters, vol. 50, 2003, pp 183-193.

[26] Y. Yamomoto, "New approach to sampled-data control systems - A function space method", Proc. 29th Conf. on Decision and Control, 1990, Honolulu, HW, pp. 1882-1887.

[27] M. Yu, L. Wang and T. Chu, "An LMI approach to networked control systems with data packet dropout and transmission delays", Mathematical Theory Networks and Systems, 2004, Leuven. 